\title{
Heart Sound Analysis in Individuals Supported With Left Ventricular Assist Device: A First Look
}

\author{
Xinlin J Chen ${ }^{1}$, Leslie M Collins ${ }^{1}$, Priyesh A Patel ${ }^{2}$, Ravi Karra ${ }^{3}$, Boyla O Mainsah ${ }^{1}$ \\ ${ }^{1}$ Duke University, Durham, North Carolina, USA \\ ${ }^{2}$ Sanger Heart and Vascular Institute, Atrium Health, Charlotte, North Carolina, USA \\ ${ }^{3}$ Duke University Medical Center, Durham, North Carolina, USA
}

\begin{abstract}
The left ventricular assist device (LVAD) has emerged as a bridge or alternative to heart transplant in individuals with advanced heart failure. However, the LVAD recipient population currently faces high rehospitalization rates. Remote analysis of precordial sounds in LVAD recipients may improve aftercare through early detection of complications. Prior work on analyses of precordial sounds in LVAD recipients focused on identifying pump thrombosis. Here, we focus on analyzing intrinsic precordial sounds to provide insight into intrinsic cardiac function. We analyzed a dataset of patient-acquired recordings of precordial sounds in $L V A D$ recipients. We developed a signal processing pipeline to separate LVAD-generated sounds from other precordial sounds, making heart sound analysis in LVAD recipients feasible. Unsupervised clustering of features extracted from LVAD-mitigated sounds revealed subgroups of subjects possessing heart sounds with distinct frequency characteristics. The results provide preliminary evidence for the potential utility of exploring heart sound analysis in LVAD recipients for remote monitoring.
\end{abstract}

\section{Introduction}

Left ventricular assist devices (LVADs) are mechanical circulatory support devices used to treat individuals with end-stage heart failure. LVAD recipients experience high rates of unplanned hospital admissions due to complications, such as heart failure, volume overload, gastrointestinal bleeding, and pump thrombosis [1]. Frequent follow-up care of LVAD recipients is one approach to mitigate complications. However, it is burdensome for LVAD recipients to conduct frequent clinical visits, particularly as they often live far away from their implanting centers [2]. This motivates the integration of remote monitoring methods into the clinical management of LVADs.
Acoustic analysis is emerging as a method to remotely and non-invasively monitor LVAD recipients. Precordial sounds in LVAD recipients are a mixture of intrinsic precordial sounds, such as heart and lung sounds, and pump-related sounds. All running pumps, the LVAD and heart included, generate characteristic acoustic signatures based on structure and loading conditions. We and others have analyzed precordial sounds in LVAD recipients and shown that differences in acoustic signatures can be used to identify thrombus formation in LVADs $[3,4]$. Furthermore, heart sounds can reveal events in the cardiac cycle, such as heart valve closures and murmurs [5], events that often inform clinicians about LVAD performance. Thus, precordial sounds may provide clinically useful and remotely obtainable information regarding cardiac function and LVAD support.

In recent years, automatic detection of abnormal heart sounds in individuals without LVADs has been widely researched [6]. However, in the case of LVAD recipients, the dominance of LVAD-generated sounds has made assessment of native heart sounds during auscultation challenging. To our knowledge, analysis of non-LVAD generated precordial sounds in LVAD recipients has not yet been attempted. Here, we leveraged knowledge of pump structure and applied signal processing methods to remove LVAD-generated sounds from LVAD recipients' precordial sounds. We analyzed time-frequency characteristics of the resulting LVAD-mitigated sounds in LVAD recipients and present results which show clusters of heart sounds with different frequency characteristics.

\section{Methods}

Signal processing and analysis were performed using MATLABC (The MathWorks, Inc.).

\subsection{Data Description}

Twenty-four patients were recruited at Duke University Hospital for a study approved by the Duke Health Institutional Review Board. Sixteen subjects had a 
HeartMate 3 (HM3; Abbott, St. Paul, MN) and eight subjects had a HeartWare VAD (HVAD; Medtronic, Minneapolis, MN). The LVAD pump speeds ranged from 5,100-6,850 revolutions per minute (rpm) for HM3s and 2,840-3,200 rpm for HVADs. Subjects recorded precordial sounds from their left upper sternal border using a Thinklabs digital stethoscope for about 1 minute, with a sampling frequency of $48,000 \mathrm{~Hz}$.

\subsection{Signal Processing}

\subsubsection{Pre-processing}

As native heart sounds are the focus of this analysis, the recordings were bandpass filtered to $20-300 \mathrm{~Hz}$ [7] and resampled at $600 \mathrm{~Hz}$.

\subsubsection{Signal Separation}

LVAD sounds were isolated from intrinsic sounds based on the acoustic energy generated by the pump during operation. The rotation of impeller blades in the LVAD produces sounds at the speed of rotation, which corresponds to the pump's fundamental frequency, and at multiples, or harmonics, of this frequency. Key LVAD harmonics are those corresponding to the blade passing frequency and its harmonics [8]:

$$
f_{n b}=f \times N \times n, \quad n=1,2, \ldots
$$

where $f_{n b}$ is the $n^{\text {th }}$ harmonic of the blade passing frequency; $f$ is the pump's fundamental frequency; $N$ is the number of impeller blades; and $n$ is a positive integer.

The HM3 and HVAD have four impeller blades [9, 10]. The HVAD is programmed to operate at a set, constant pump speed. In contrast, the HM3 employs an 'artificial pulse' feature and is programmed to undergo speed changes every two seconds from a set speed: dropping by $2,000 \mathrm{rpm}$ for 0.15 seconds (s) and

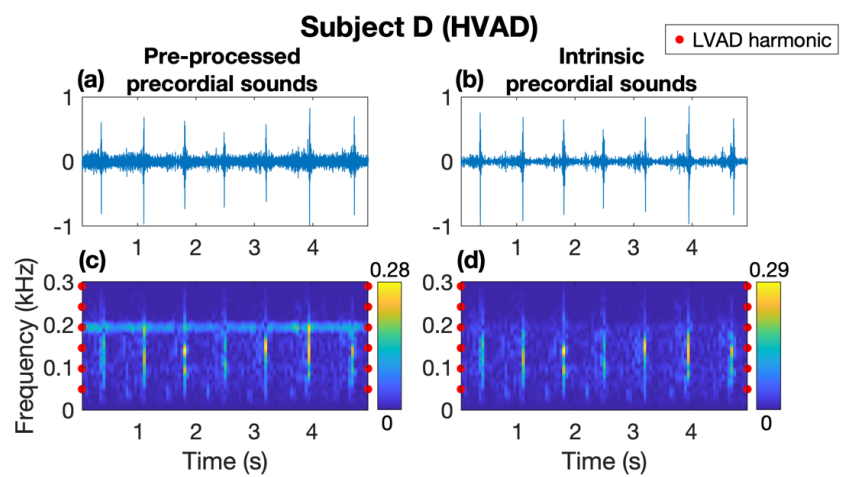

increasing by $4,000 \mathrm{rpm}$ for $0.2 \mathrm{~s}$ before returning to the set speed [10].

Adaptive noise cancellation, employing the normalized least mean squares (nLMS) algorithm, was applied to separate the pre-processed precordial sounds into LVADgenerated sounds and intrinsic sounds. A noise reference was used to adaptively estimate the contributions of the LVAD sounds in the precordial sound mixture, which was then subtracted from the precordial sounds to obtain an estimate of intrinsic sounds, or LVAD-mitigated sounds. The frequency spectrum of each subject's LVAD sounds was determined by the pump speed and structure, knowledge of which was used to generate simulated noise references. The step-size of the adaptive filter was empirically chosen for each recording by balancing the attenuation of LVAD harmonics and preservation of the background power spectrum around LVAD harmonics.

\subsection{Unsupervised Clustering}

Acoustic features were extracted from both preprocessed precordial sounds and LVAD-mitigated sounds to identify similarities in acoustic patterns between subjects. The acoustic features consisted of Welch's power spectral densities (PSDs) estimated from five $5 \mathrm{~s}$ segments from each recording, using a Hanning window length of 200 milliseconds (ms) and an overlap of 100 $\mathrm{ms}$; the 5-second time window was chosen to represent the frequency content of each subject's precordial sounds, while accounting for potential signal variability over time. Uniform Manifold Approximation and Projection (UMAP) [11] was used to perform manifold learningbased dimensionality reduction of acoustic features. PSDs were used as inputs to the UMAP algorithm with a similarity metric of correlation and a local neighborhood size constrained to 10 neighbors.

\section{Results}

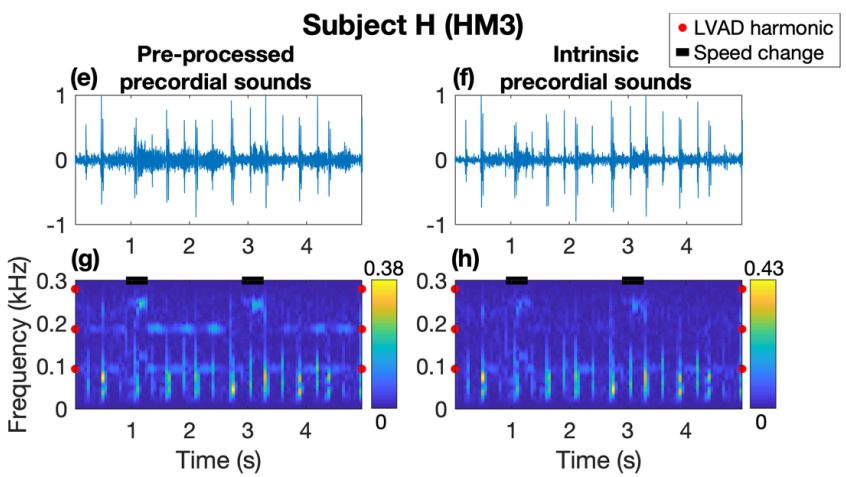

Figure 1. Effect of adaptive filtering on LVAD noise in precordial sounds from subjects D and H, a HeartWare VAD and HeartMate 3 (HM3) recipient, respectively. Time domain signals (top) and spectrograms (bottom) of (a, c) pre-processed precordial sounds and (b, d) intrinsic sounds from subject $\mathrm{D}$; and $(\mathrm{e}, \mathrm{g})$ pre-processed precordial sounds and $(\mathrm{f}, \mathrm{h})$ intrinsic sounds from subject $\mathrm{H}$. Spectrograms were generated with a Hanning window length of $100 \mathrm{~ms}$ and overlap of $50 \mathrm{~ms}$. Harmonics of the frequency corresponding to the set pump speed are annotated with a red dot. Times of speed changes in the HM3 are annotated with a black horizontal line. 

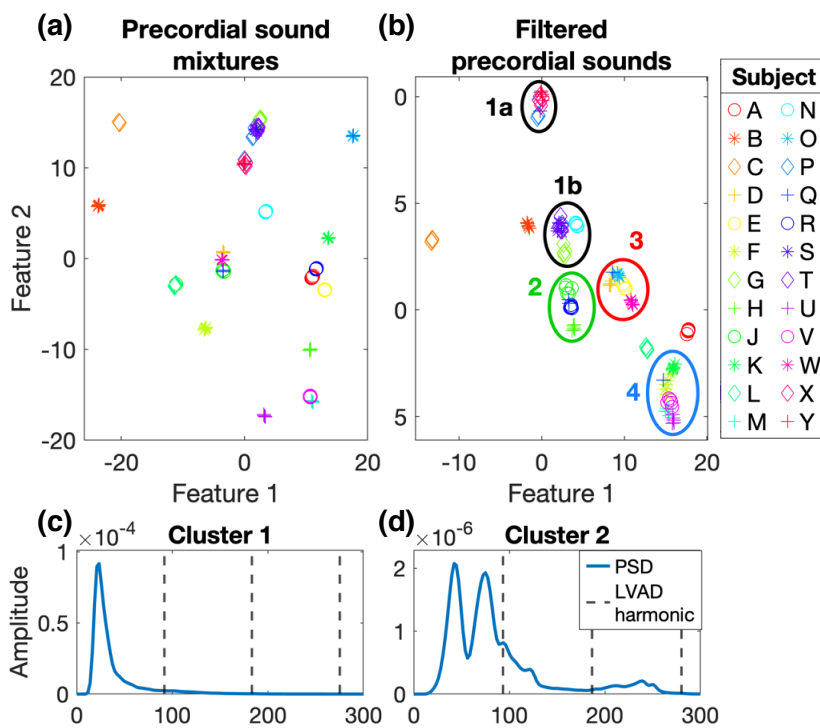

(d) $\times 10^{-6} \quad$ Cluster 2
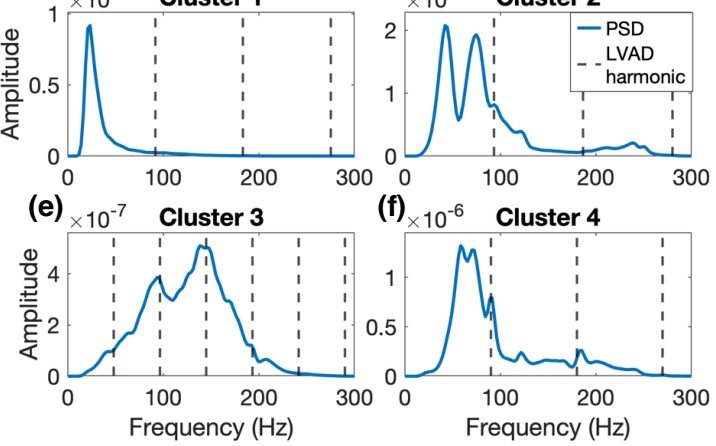

Figure 2. Uniform Manifold Approximation and Projection visualization of power spectral densities (PSDs) calculated from (a) pre-processed precordial sounds, and (b) LVAD-mitigated sounds. (c-f) Examples of representative PSDs from the clusters of LVAD-mitigated sounds in (b), with harmonics of the primary pump speed indicated, from: (c) subject X, (d) subject $H$, (e) subject D, and (f) subject F.

Spectrograms of pre-processed precordial sounds and LVAD-mitigated sounds from one HM3 and one HVAD recipient appear in Figure 1a-b and c-d, respectively. Strong contributions from LVAD harmonics are evident in the spectrograms of pre-processed precordial sounds; for the HM3, artificial pulse regions are indicated. The spectrograms of LVAD-mitigated sounds show pronounced attenuation of LVAD harmonics with adaptive filtering (Figure $1 \mathrm{~b}$ and $\mathrm{d}$ ). Short energy bursts below $250 \mathrm{~Hz}$ in frequency, which correspond to the expected frequency range and duration $(<0.15 \mathrm{~s})$ of heart sounds $[7,12,13]$ do not appear to have been impacted by adaptive filtering.

UMAP transformations of power spectral features of pre-processed precordial sounds and LVAD-mitigated sounds are shown in Figure 2a and 2b, respectively. The clustering of pre-processed precordial sounds was driven by LVAD-generated frequencies. Isolated subject-specific clusters can be observed, as most of the subjects had a unique pump speed. After adaptive filtering was used to remove LVAD-generated sounds, clustering was driven by biological sounds and equipment-related noise, with representative power spectra shown in Figure 2c-f.

Cluster $\mathbf{1}$ is generally characterized by sounds with dominant low-frequency components from sub-50 Hz noise (Figure 2c), which is more prominent in cluster 1a. From our experience, this noise is generated when pressure is applied to the stethoscope. Prior to bandpass filtering, the noise peaked between $4.5-14.0 \mathrm{~Hz}$.

Cluster 2 is characterized by low frequency sounds with bimodal peaks below $100 \mathrm{~Hz}$ (Figure 2d). Cluster members include subjects $H, R$, J, and Q. LVADmitigated sounds from Subject $\mathrm{H}$ (Figure 1h) are characterized by short, low-frequency energy bursts with a frequency band between $25-120 \mathrm{~Hz}$, suggesting that these bimodal peaks result from heart sounds $[7,12,13]$.

Cluster 3 is characterized by sounds with a wide frequency band (Figure 2e). Cluster members include subjects D, E, O, and W. Subjects O and W's LVADmitigated sounds appear to be dominated by lung sounds: energy bursts with frequency components from 50-200 $\mathrm{Hz}$ [14] lasting about 1s. LVAD-mitigated sounds from subjects D and $\mathrm{E}$ fit heart sound time-frequency characteristics $[12,13]$, though they differ from those in Cluster 2. In subject D's LVAD-mitigated sounds (Figure 1d), short, high-intensity bursts with a frequency band of $50-200 \mathrm{~Hz}$ occur about every $0.7 \mathrm{~s}$. Fainter, short energy bursts with a frequency band of $25-60 \mathrm{~Hz}$ precede the high-intensity bursts by about $0.3 \mathrm{~s}$, suggesting a weaker heart sound.

Cluster 4 is characterized by low frequency sounds below $100 \mathrm{~Hz}$, with a peak around $75 \mathrm{~Hz}$ (Figure 2f). All subjects in this cluster (F, K, M, U, V) had an HM3 LVAD. Their sounds contained 'triple' energy bursts that were synchronized with the timing of the artificial pulse (Figure 3a-b) but did not correspond to LVAD frequencies at those times; this suggests that the triple peaks are unlikely to be generated by the LVAD and are possibly heart sound-related.

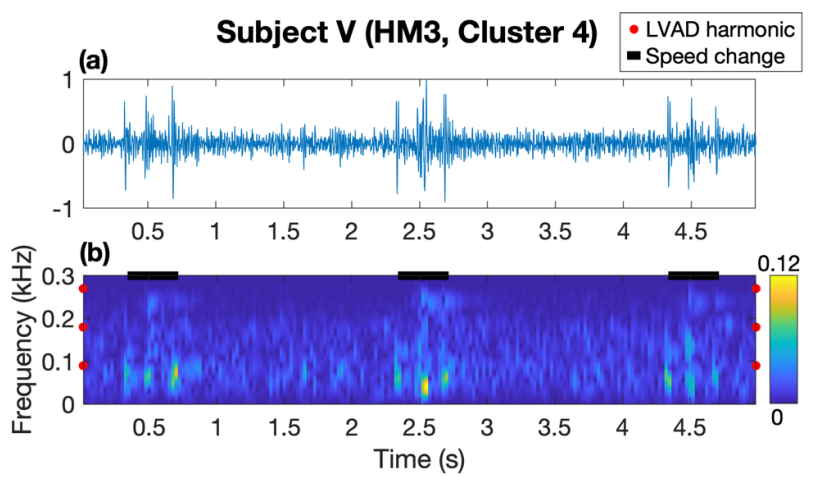

Figure 3. Time domain signal (top) and spectrogram (bottom) of LVAD-mitigated sounds from subject V, a HeartMate 3 (HM3) recipient within cluster 4 in Figure 2b. A Hanning window of 50 $\mathrm{ms}$ and overlap of $25 \mathrm{~ms}$ were used to generate the spectrogram. Harmonics of the frequency corresponding to the set pump speed are annotated with a red dot. Speed changes arising from the HM3's artificial pulse are annotated with a black horizontal line. 
In summary, our analysis of LVAD-mitigated sounds in LVAD recipients revealed various acoustic signatures that may be related to innate cardiac function and may therefore be of clinical interest.

\section{Conclusion}

Previous studies on acoustic analysis of precordial sounds in LVAD recipients have focused on monitoring pump function. To our knowledge, this is the first analysis of intrinsic precordial sounds in LVAD recipients, made feasible via adaptive noise cancellation of LVAD harmonics. Heart sounds may reveal information about events in the cardiac cycle; our unsupervised analysis of LVAD-mitigated sounds in LVAD recipients revealed clusters of subjects with different precordial frequency characteristics, which may be associable with cardiac function. These findings provide support for the potential utility of remotely monitoring cardiac activity in LVAD recipients via analysis of their intrinsic precordial sounds. We will explore the clinical significance of these findings in future work.

\section{Acknowledgments}

This study was funded by a grant from the Duke Institute for Health Innovation. The authors would like to thank Clive Smith and Thinklabs Medical LLC for providing stethoscopes for the study.

\section{References}

[1] N. A. Haglund, M. E. Davis, N. M. Tricarico, M. E. Keebler, and S. Maltais, "Readmissions After Continuous Flow Left Ventricular Assist Device Implantation: Differences Observed Between Two Contemporary Device Types," ASAIO J, vol. 61, no. 4, pp. 410-6, Jul-Aug 2015.

[2] J. L. Cook et al., "Recommendations for the Use of Mechanical Circulatory Support: Ambulatory and Community Patient Care: A Scientific Statement From the American Heart Association," Circulation, vol. 135, no. 25, pp. e1145-e1158, 2017.

[3] G. L. Yost, T. J. Royston, G. Bhat, and A. J. Tatooles,
"Acoustic Characterization of Axial Flow Left Ventricular Assist Device Operation In Vitro and In Vivo," ASAIO J., vol. 62, no. 1, pp. 46-55, Jan-Feb 2016.

[4] P. Patel, B. Mainsah, C. A. Milano, D. P. Nowacek, L. Collins, and R. Karra, "Acoustic Signatures of Left Ventricular Assist Device Thrombosis," ASME J. of Medical Diagnostics, vol. 2, no. 2, 2019.

[5] J. S. Coviello, "Section 1: Heart sound fundamentals," in Auscultation skills: breath \& heart sounds, 5th ed. Philadelphia, PA, US: Lippincott Williams \& Wilkins, p. 7.

[6] A. K. Dwivedi, S. A. Imtiaz, and E. Rodriguez-Villegas, "Algorithms for Automatic Analysis and Classification of Heart Sounds-A Systematic Review," IEEE Access, vol. 7, pp. 83168345, 2019.

[7] P. J. Arnott, G. W. Pfeiffer, and M. E. Tavel, "Spectral Analysis of Heart Sounds: Relationships Between Some Physical Characteristics and Frequency Spectra of First and Second Heart Sounds in Normals and Hypertensives," $J$. Biomed. Eng., vol. 6, no. 2, pp. 121-128, Apr. 1984.

[8] M. P. Norton and D. G. Karczub, "Noise and vibration as a diagnostic tool," in Fundamentals of Noise and Vibration Analysis for Engineers, 2nd ed. Cambridge, UK: Cambridge University Press, 2003, p. 524.

[9] HeartWare, Inc., "Principles of Operation," HeartWare Ventricular Assist System: Instructions for Use. Miami Lakes, FL: HeartWare, Inc., 2012.

[10] L. Wiegmann et al., "Fluid Dynamics in the HeartMate 3: Influence of the Artificial Pulse Feature and Residual Cardiac Pulsation," Artificial Organs, vol. 43, no. 4, pp. 363-376, 2019.

[11] L. McInnes, J. Healy, and J. Melville, "UMAP: Uniform Manifold Approximation and Projection for Dimension Reduction," arXiv, p. 1802.03426

[12] C. J. Wiggers and J. Archie L. Dean, "The Nature and Time Relations of the Fundamental Heart Sounds," Am. J. Physiol.Legacy Content, vol. 42, no. 3, pp. 476-497, 1917.

[13] S. McGee, "Chapter 39 - Auscultation of the Heart: General Principles," in Evidence-Based Physical Diagnosis, 4th ed. Philadelphia, PA, USA: Elsevier, 2018, pp. 327-332.

[14] M. Sarkar, I. Madabhavi, N. Niranjan, and M. Dogra, "Auscultation of the Respiratory System," Ann. Thorac. Med., vol. 10, no. 3, pp. 158-68, Jul-Sep 2015.

Address for correspondence:

Boyla Mainsah, Ph.D.

Pratt School of Engineering, Duke University boyla.mainsah@duke.edu 